\title{
CORRECTION
}

\section{Correction: Antibiotic-induced microbiome perturbations are associated with significant alterations to colonic mucosal immunity in rhesus macaques}

Jennifer A. Manuzak, Alexander S. Zevin, Ryan Cheu, Brian Richardson, Jacob Modesitt, Tiffany Hensley-McBain, Charlene Miller, Andrew T. Gustin, Ernesto Coronado, Toni Gott, Mike Fang, Michael Cartwright, Solomon Wangari, Brian Agricola, Drew May, Elise Smith, Hans Benjamin Hampel, Michael Gale, Cheryl M. Cameron, Mark J. Cameron, Jeremy Smedley and Nichole R. Klatt Mucosal Immunology (2020) 13:558; https://doi.org/10.1038/s41385-020-0274-x

Correction to: Mucosal Immunology https://doi.org/10.1038/ s41385-019-0238-1, published online 4 December 2019

The original version of this Article was updated shortly after publication, because some accession numbers for the datasets were inadvertently omitted.
Accession numbers for the datasets are GSE143729 and PRJNA604177.

This error has now been corrected in both the PDF and HTML versions of the Article. 